\title{
Neoadjuvant Systemic Therapy in Breast Cancer
}

\author{
Vladimir F. Semiglazov ${ }^{1}$ and Vladislav V. Semiglazov ${ }^{2}$ \\ 1 Petrov Research Institute of Oncology, St. Petersburg \\ ${ }^{2}$ St.-Petersburg Pavlov Capital Medical University \\ Russia
}

\section{Introduction}

Neoadjuvant systemic therapy (NST) has become a frequently used option for systemic therapy in primary operable breast cancer. All patients with a clear indication for adjuvant systemic treatment can be offered systemic therapy preoperatively. These recommendations focus on early response to NST and on tailoring therapy to response and biological and histological markers.

Three main goals for NST in operable breast cancer were defined:

- $\quad$ To reduce mortality from breast cancer with reduced toxicity.

- To improve surgical options.

- To acquire early information on response and biology of the disease.

A recent Oxford meta-analysis (EBCTCG, 2005) of randomized studies of more than 4000 women, comparing postoperative and neoadjuvant chemotherapy for operable breast cancer, demonstrated equivalent overall survival rates with a hazard ratio of $0.98(p=0.67)$. Neoadjuvant chemotherapy was associated with fewer adverse effects, and associated with a higher rate of breast conserving surgery $(\mathrm{p}<0.001)$. In addition, patients who achieved a pCR had a better survival than those who had residual disease in the breast and lymph nodes. Neoadjuvant chemotherapy is associated with a small increase in the risk of locoregional recurrence in patients who went on to receive radiotherapy without surgery as local therapy.

\section{Neoadjuvant systemic therapy}

\subsection{Neoadjuvant chemotherapy}

Some early nonrandomized and randomized trials suggested that neoadjuvant chemotherapy might result in improved disease-free survival rates compared with standard adjuvant treatment (Scholl et al., 1994; Semiglazov et al. 1994), but some of these trials were not designed as a direct comparison of preoperative and postoperative chemotherapy. In 1998, the National Surgical Adjuvant Breast and Bowel Project (NSABP) reported the result of a large prospective randomized trial (Protocol B- 18) that compared 4 cycles of doxorubicin and cyclophosphamide (AC) given preoperatively to the same dose of AC given postoperatively (Fisher et al., 1998; Wolmark et al., 2001). The disease-free survival and overall survival rates for the 2 treatment arms of this trial were almost identical. B-18 
demonstrated that clinical and pathologic tumor response were predictors of overall survival. Similar to other reports, despite a $36 \%$ clinically complete response (cCR) rate, only $13 \%$ of all patients had a pathologically complete response (pCR), defined as the absence of invasive tumor in the breast. A meta-analysis of 9 randomized studies (not involving taxanes) demonstrated the equivalence of neoadjuvant and adjuvant treatments for breast cancer in terms of survival, disease progression, and distant recurrence and showed that an increased risk of locoregional disease recurrence is associated with neoadjuvant treatment, especially when primary systemic treatment is not accompanied by any surgical intervention (eg, radiation therapy alone) (Mauri et al., 2005).

Preoperative neoadjuvant chemotherapy with agents such as doxorubicin and taxanes is an effective treatment for patients with breast cancer and leads to an increased rate of successful breast- conserving surgery and a decreased proportion of patients with metastatic involvement of the axillary lymph nodes (Kaufmann et al., 2006). Neoadjuvant chemotherapy also provides an opportunity to assess potential responses of the tumor to a given agent, which is an important consideration in selecting postoperative (adjuvant) therapy. Data from large phase 2 and phase 3 chemotherapy trials have shown that 3 to 4 months of preoperative treatment can be given without compromising either locoregional control or long-term survival (Bonadonna et al., 1998; Smith et al., 2002).

The NSABP Protocol B-27 was designed to determine the effect of adding docetaxel after 4 cycles of preoperative doxorubicin and cyclophosphamide on clinical and pathological response rates and on disease-free survival and overall survival of women with operable breast cancer. There were trends toward improved disease-free survival with the addition of docetaxel. Preoperative docetaxel, but not postoperative docetaxel, significantly improved disease-free survival in patients who had a clinical partial response after doxorubicin and cyclophosphamide. Pathologic complete response, which was doubled (from 13\% to 26\%) with preoperative docetaxel, was a significant predictor of overall survival regardless of treatment (Bear et al., 2006).

European Cooperative Trial in Operable Breast Cancer (ECTO) was designed to assess the effects of adding paclitaxel to an anthracycline- based regimen in patients with operable breast cancer, and to compare the same regimen given preoperatively and postoperatively (Gianni et al., 2009).

The ECTO study found a significant improvement in distant recurrence free survival (DRFS) in patients with operable early-stage breast cancer when paclitaxel was incorporated into a sequential adjuvant regimen of noncross-resistant chemotherapies that was originally pioneered by the Milan group (Gianni et al., 2009). This advantage was also seen in women with node-negative disease who constituted $40 \%$ of patients enrolled in the adjuvant arms. Comparison of the same paditaxel/doxorubicin/CMF regimen given preoperatively instead of postoperatively resulted in similar DRFS but a significantly higher percentage of patients were able to undergo breast-conserving surgery without a detrimental effect on local recurrence or survival.

The ECTO study recruited a typical and representative sample of patients and its findings are consistent with a recent meta-analysis from the Early Breast Cancer Trialists Group, which showed that taxane-based adjuvant regimens are superior to anthracycline-based regimens in terms of recurrence rate (Peto, 2007). Pooled data from another meta-analysis also showed that incorporation of taxanes into anthracycline-based regimens significantly improved both disease-free (DFS) and overall survival (OS) in patients with early-stage breast cancer (De Laurentiis et al., 2008). 


\subsection{Duration and sequence of neoadjuvant chemotherapy}

The superior outcomes of patients who achieved favorable responses in the breast had led investigators to question whether using in-breast response as an in vivo chemosensitivity test and tailoring therapy accordingly may improve outcomes. GeparTrio was one of the studies that set out to answer this question. In this multicenter German study, all 2,090 patients received an initial 2 cycles of neoadjuvant TAC chemotherapy (docetaxel $75 \mathrm{mg} / \mathrm{m}^{2}$, doxorubicin $50 \mathrm{mg} / \mathrm{m}^{2}$, and cyclophosphamide $500 \mathrm{mg} / \mathrm{m}^{2}$ every 21 days). Patients were then divided on the basis of sonographic evaluation into responders (tumor size decreased by $>50 \%$ ) and nonresponders (tumor size decreased by $<50 \%$ ). A third group, patients whose tumors increased in size by $25 \%$ or more, was removed from the study and treated at the discretion of their oncologist. The study continued in two parts, one evaluating a change of therapy for nonresponders, and one evaluating the optimal duration of therapy in the responders (Von Minckwitz et al., 2008).

In the first part, the 622 patients who did not respond to the initial 2 cycles of TAC chemotherapy were randomly assigned to four more cycles of TAC chemotherapy or four 21-day cycles of an NX regimen (vinorelbine $25 \mathrm{mg} / \mathrm{m}^{2}$ on days 1 and 8 and capecitabine [Xeloda] $1,000 \mathrm{mg} / \mathrm{m}^{2}$ orally twice daily on days $1-14$ ). Sonographic response rate was chosen as the primary endpoint, and it should be noted that the statistical plan was based on a hypothesis of non-inferiority (rather than superiority) of NX compared to TAC. There was no difference in sonographic response rates for the two regimens, confirming the non-inferiority of NX. The rates of pCR were low for both NX and TAC, at $6.0 \%$ and $5.3 \%$, respectively. It must be emphasized that this study did not set out to demonstrate an improvement in outcome for switching to a non- cross-resistant chemotherapy regimen, nor did it show such a difference. In the second part of the GeparTrio study, the 1,390 patients who responded to an initial 2 cycles of neoadjuvant TAC chemotherapy were randomized to either 4 or 6 further cycles of TAC pre-operatively, ie, 6 versus 8 cycles in total. The primary aim of this part of the study was to detect an increased pCR rate of $26 \%$ versus $20 \%$ in the I group receiving a longer duration of therapy. There were no significant differences in the rates of pCR ( 8 cycles $23.5 \%$ vs 6 cycles $21.0 \%, \mathrm{P}=0.27$ ) or BCS $(67.5 \%$ vs $68.5 \%, P=.68$ ) (von Minckwitz et al., 2008). Thus, the knowledge of chemotherapy sensitivity does not appear to predict a greater benefit for more of what was already proven effective (TAC, in this case).

The Aberdeen study also assessed the potential benefit of switching chemotherapy regimens in the neoadjuvant setting, but in this case the randomization between "sticking or switching" occurred in the responders rather than the nonresponders (Smith et al., 2002). In this study, 162 patients were enrolled and received four 21-day cycles of an anthracycline chemotherapy regimen (CVAP: cyclophosphamide 1,000 mg/m², vincristine $1.5 \mathrm{mg} / \mathrm{m}^{2}$, doxorubicin $50 \mathrm{mg} / \mathrm{m}^{2}$, and prednisolone $40 \mathrm{mg}$ for 5 days). The 104 patients classified as responders by clinical assessment were randomized to 4 cycles of CVAP or 4 cycles of docetaxel (100 mg/m² every 21 days). All 55 nonresponders received 4 cycles of docetaxel. Intention-to-treat (ITT) analysis showed that the addition of docetaxel significantly enhanced cRR in the responders, compared to continuation of CVAP ( $85 \%$ vs $64 \%, P=0.03)$. The pCR rate was also superior in the docetaxel group (ITT analysis, $31 \%$ vs $15 \%, \mathrm{P}=0.06$; for patients completing 8 cycles, $34 \%$ vs $16 \%, \mathrm{P}=0.04$ ). In addition, updated follow-up at 3 years indicated improved survival in the docetaxel arm, although this was not a primary endpoint of the study design and was not incorporated into statistical plan (Heys et al., 
2002). However, nonresponders also benefited from switching to docetaxel, with over half $(55 \%)$ of these patients going on to achieve clinical responses, and a small proportion ( $2 \%)$ achieving pCRs. Neither the GeparTrio nor the Aberdeen studies therefore provide evidence of a convincing role for response (or lack thereof) to neoadjuvant chemotherapy as an in vivo tool for chemotherapy selection, but they suggest that most patients may benefit from exposure to a varied chemotherapy approach in the neoadjuvant setting.

\subsection{Neoadjuvant endocrine therapy}

A useful strategy to improve knowledge about treatment effects is the early identification of features, which are associated with response or resistance to primary therapy. Previously published studies indicated that pathological complete remission (pCR) rate was significantly higher following preoperative chemotherapy for patients whose tumors did not express estrogen receptor (ER) and progesterone receptor (PgR), compared with the receptor- positive cohort (Ring et al., 2004; Colleoni et al., 2004). Despite the significantly higher incidence of pCR achieved by preoperative chemotherapy for patients with endocrine-nonresponsive disease, the disease-free survival (DFS) was significantly worse for this cohort compared with the ER positive expression cohort in several studies (Colleoni et al., 2008).

More recently neoadjuvant endocrine therapy has emerged as an attractive alternative in postmenopausal women with large or inoperable hormone receptor positive breast cancers. Although there have been no large randomized trials comparing surgery with neoadjuvant endocrine therapy, there have been a series of studies using aromatase inhibitors (AIs) which have produced promising results. A number of large randomized trials have compared various AIs directly with tamoxifen. An important endpoint in each of these studies has been the rate at which breast conservation has been achieved. There are a number of benefits to using neoadjuvant therapy compared with primary surgery. The most obvious benefit is that women with large operable or locally advanced breast cancers can be downstaged allowing them to become operable or more suitable for less extensive surgery (Dixon \& Macaskill, 2009). For instance, those who originally would have required mastectomy can often be converted to breast-conserving surgery. This is an advantage because studies have demonstrated that breast-conserving surgery followed by radiotherapy has significant psychological benefits, better cosmetic outcomes, and comparable disease control rates compared to mastectomy. There are as yet limited longterm data on patients who have had breast-conserving surgery after neoadjuvant therapy, but the results to date are reassuring. The majority of patients who are spared mastectomy with neoadjuvant endocrine therapy are elderly, but studies have shown that even in older women, if they are given the choice, they are no more likely to choose mastectomy than younger women. Neoadjuvant endocrine therapy is also an excellent treatment for older patients with estrogen receptor cancers who are unfit for surgery because of significant comorbidities. For these patients, shrinkage can allow resection under local anesthesia, or for a select group with short life expectancy, treatment with endocrine therapy can provide long-term disease control for the rest of their lives.

\subsubsection{Letrozole compared with tamoxifen}

The first endocrine neoadjuvant study was the P024 trial and included 337 postmenopausal women with large operable or locally advanced ER-positive and PR-positive breast cancers (Eiermann et al., 2001). All patients required mastectomy at diagnosis or were inoperable. In 
this study patients were randomly selected to receive 4 months of letrozole or 4 months of tamoxifen. Objective response rates (ORR) by palpation, mammography, and ultrasound were all significantly higher in the letrozole treated group. There was also a significantly higher rate of breast-conserving surgery for patients randomly assigned to receive letrozole $(45 \%$ vs. $35 \%$ in the tamoxifen group; $\mathrm{p}=0.022)$.

\subsubsection{Anastrozole compared with tamoxifen}

Two large randomized studies have compared anastrozole with tamoxifen. In the Immediate Preoperative Arimidex, Tamoxifen or Combined with Tamoxifen (IMPACT) trial, 330 patients from the UK and Germany were randomly selected to receive anastrozole alone, tamoxifen alone, or a combination for 3 months before surgery (Smith et al., 2005). The study differed from $\mathrm{P} 024$ in that patients who were suitable for breast-conserving surgery at the outset were enrolled. There was no significant difference seen in ORRs between the three treatments as measured by calipers and ultrasound. There was a subgroup of 124 patients who were considered to require mastectomy at baseline. Although there remained no difference in this group in ORR, a significantly higher number of women were deemed suitable for breast-conserving surgery following treatment with anastrozole, compared with tamoxifen ( $46 \%$ vs. $22 \%$; $p=0.03$ ).

In the Preoperative Arimidex Compared with Tamoxifen trial, the entry criteria was similar to the IMPACT trial, although this study also included patients who were inoperable (Cataliotti et al., 2006). This study also differed in that it included a group of patients who were given concurrent neoadjuvant chemotherapy. Randomisation was to the 202 patients treated with anastrozole alone or the 201 patients treated with tamoxifen alone for 3 months. There was no significant difference in ORR by ultrasound or caliper measurements between the different treatment arms, although there was a trend in favor of anastrozole for those patients treated with neoadjuvant endocrine therapy alone. There was a significantly higher ORR in the anastrozole group for patients whose tumors were initially assessed as requiring mastectomy or were inoperable.

A combined analysis of the two anastrozole studies included 535 patients and again failed to show any difference between treatments (Smith, 2004). There was again an overall improvement in ORR in favor of anastrozole in the subgroup of patients who were deemed to require mastectomy or be inoperable at the outset. Both were assessed by calipers $(47 \%$ vs. $35 \% ; p=0,026)$ and ultrasound $(36 \%$ vs. $26 \% ; p=0.048)$. A significant change in both feasible and actual surgery in favor of anastrozole was also evident for those patients who required a mastectomy or were inoperable at diagnosis.

\subsubsection{Exemestane compared with tamoxifen}

Several recent studies support the use of aromatase inhibitors as neoadjuvant therapy for hormone-responsive breast cancer. For example, we reported the results of a study comparing the efficacy of exemestane and tamoxifen as neoadjuvant therapy (Semiglazov et al., 2005). In that study, 151 postmenopausal women with ER-positive and/or PgR-positive breast cancer were randomly assigned to receive exemestane or tamoxifen for 3 months. Neoadjuvant treatment with exemestane significantly improved clinical objective response ( $76 \%$ vs $40 \% ; \mathrm{P}=.05$ ) and the rate of breast-conserving surgery ( $37 \%$ vs $20 \%$; $=0.05)$, but it did not result in any significant differences in objective response as determined by mammogram or ultrasound. Thus, exemestane is more effective than tamoxifen as a neoadjuvant treatment option for postmenopausal women with ER-positive disease. 


\subsubsection{Hormonal versus chemotherapy in the neoadjuvant treatment}

Duration of neoadjuvant hormonal treatment for breast cancer in most studies was 3-6 months. The few studies that investigated prolonged treatment with neoadjuvant endocrine therapy suggest that a further reduction in tumour size can be achieved and that even surgery can be withheld for elderly women on continuing hormonal treatment. However, the optimum duration of neoadjuvant endocrine therapy has to be established.

For many years, primary systemic (neoadjuvant) therapy has been given before local treatment for women with locally advanced breast cancer in an effort to make such disease operable. Chemotherapy has been the mainstay of this approach, but more recently neoadjuvant endocrine therapy has emerged as an attractive alternative in post-menopausal women with large hormone receptor positive breast cancers. A number of randomized trials (like P024, IMPACT, PROACT) have compared various aromatase inhibitors directly with tamoxifen. An important endpoint in each of these studies has been the rate at which breast conservation has been achieved. The presence of steroid hormone receptors (ER and/or PR) are target for endocrine therapy. Preoperative chemotherapy may be less effective in postmenopausal patients with ER-positive and/or PR-positive tumors at least with respect to doxorubicin-containing or taxane-containing regimens. Pathological complete response (pCR) rates after chemotherapy were significantly higher among patients with tumors that were both ER-negative and PR-negative compared with patients whose tumors had any (even low) expression of steroid hormone receptors (Colleoni et al. 2004, 2008). In the ECTO I trial, pCR after neoadjuvant chemotherapy was observed in $42 \%$ of women with ERnegative tumors, compared with $12 \%$ in the ER-positive group (Gianni et al. 2009). In the NSABP B-27 study, ER-negative tumors had higher rates of pCR than ER-positive tumors when treated with neoadjuvant $\mathrm{AC}$, as well as when treated with AC followed by docetaxel (Bear ., et al. 2006). Before our trial there were few, if any, direct comparisons of primary neoadjuvant endocrine therapy with primary neoadjuvant chemotherapy in patients with hormone-responsive breast cancer.

This was an open-label, randomized phase 2 trial of once-daily endocrine therapy (exemestane or anastrozole) or chemotherapy (doxorubicin and paclitaxel, every 3 week for 4 cycles) in postmenopausal women with primary ER-positive breast cancer. A total of 239 patients with ER-positive and/or PgR-positive breast cancer (T2N1-2, T3N0-1, T4N0M0) were randomly assigned to receive neoadjuvant endocrine therapy (ET) [anastrazole 1 $\mathrm{mg}$ /day or exemestane $25 \mathrm{mg} /$ day for 3 months, 121 patients] or chemotherapy (CT) [doxorubicin $60 \mathrm{mg} / \mathrm{m} 2$ with paclitaxel $200 \mathrm{mg} / \mathrm{m} 2$, four 3-week cycles, 118 patients]. All patients were considered to be ineligible for breast-conserving surgery (BCS) at enrollment. After BCS all patients received radiotherapy (50 Gy in 25 fractions). The median follow-up time was 5.6 years.

The primary efficacy end point was already reported (Semiglazov et al., 2007). Overall response $(\mathrm{OR}=\mathrm{CR}+\mathrm{PR})$ was similar in the endocrine therapy group $(65.5 \%)$ compared with chemotherapy group $(63.6 \% ; \mathrm{p}>0.5)$.

Interim analysis of this trial showed similar objective response in patients who were receiving exemestane and in patients who were receiving anastrazole. It allowed us to review and to analyze dates on all patients who were receiving aromatase inhibitors in the endocrine therapy group.

There was a trend toward higher overall rates of OR and breast-conserving surgery among patients with tumors expressing high levels of ER (Allred score $\geq 6$ ) in the endocrine therapy compared with the chemotherapy group ( $43 \%$ vs $24 \%$, $\mathrm{p}=0.054$; Table 1 ). 


\begin{tabular}{|l|c|c|c|}
\hline & $\begin{array}{c}\text { Endocrine } \\
\text { Therapy }\end{array}$ & Chemotheapy & \\
\hline Response, $\mathrm{n}(\%)$ & $(\mathrm{n}=70)$ & $(\mathrm{n}=63)$ & P Value \\
\hline Clinical objective response & $49(70)$ & $38(60)$ & 0.068 \\
\hline Mammography & $46(66)$ & $38(60)$ & 0.088 \\
\hline Breast-conserving surgery & $30(43)$ & $15(24)$ & 0.054 \\
\hline
\end{tabular}

*High levels of estrogen receptor expression are defined as $\geq 6$ Allred score or $\geq 120 \mathrm{fmol} / \mathrm{g}$.

Table 1. Overall Objective Response in Patients With High Levels of Estrogen Receptor Expression*

After completing neoadjuvant treatment, 31 patients $(13 \%)$ did not undergo surgical resection: $12.3 \%$ of patients who were receiving endocrine therapy and $13.5 \%$ of patients who were receiving chemotherapy. Twenty-two patients did not receive surgery because of disease progression. These patients were switched to the other study therapy: patients initially treated with endocrine therapy received chemotherapy, and patients treated with chemotherapy received endocrine therapy. Progressive disease was observed in $9 \%$ of patients who were receiving endocrine therapy and $9 \%$ of patients who were receiving chemotherapy $(\mathrm{P}>0.5)$. Stable disease was seen in $21 \%$ of patients who were receiving endocrine treatment and $26 \%$ of patients who were receiving chemotherapy.

Analysis of BCS rates according to pretreatment characteristics showed a non-significant trend towards increased BCS in patients with clinical stage T2, ER+/PgR+, 70 years and older ( $\mathrm{p}=0.054-0.088$ ) receiving neoadjuvant endocrine therapy.

The rate of BCS was particularly marked in patients receiving endocrine therapy, who achieved a clinical response. There was no significant difference between endocrine therapy (ET) and chemotherapy (CT) relative to the incidence of locoregional recurrences and distant metastases $(8.2 \%$ and $7.6 \%, \mathrm{p}=0.99 ; 14.8 \%$ and $15.2 \%, \mathrm{p}=0.83$, respectively). There was no significant difference in DFS through 5 years of follow up between the 121 patients who received neoadjuvant endocrine therapy and 118 women who received chemotherapy: $71.0 \%$ and $67.7 \%(p>0.5)$. After a median follow up of 5.6 years 35 events had been reported in the endocrine group (24 in 66 patients who underwent mastectomy and 11 in 40 patients who underwent BCS). 5-year DFS was $63.6 \%$ after mastectomy and $72.5 \%$ after BCS $(p=0.076)$. The incidence of commonly reported adverse events was higher in patients receiving chemotherapy. No serious adverse events were reported in patients receiving endocrine therapy. Six patients receiving chemotherapy experienced febrile neutropenia leading to treatment interruption. No deaths occurred during the preoperative therapy.

Our trial has shown that preoperartive endocrine therapy with aromatase inhibitors offers the same rate of overall objective response, breast-conserving surgery, 5-years DFS as chemotherapy in postmenopausal patients with ER-positive tumors. The frequency of adverse events was higher among patients who were receiving chemotherapy. Endocrine treatment was well tolerated. Preoperative endocrine therapy with aromatase inhibitors is a reasonable alternative to preoperative chemotherapy for postmenopausal women with ERpositive disease in clinical situation in which the low toxicity of the regimen is considered an advantage. According St.Gallen recommendation (Goldhirsch et al., 2009) neoadjuvant 
endocrine therapy without chemotherapy was considered reasonable for postmenopausal patients with strongly receptor-positive disease. If used, such treatment should be considered for a duration of 5-8 months or until maximum tumour response.

\subsection{Neoadjuvant therapy in HER2+ breast cancer}

Amplification or overexpression, or both, of human epidermal growth factor receptor-2 (HER2, also known as ERBB2), a transmembrane receptor tyrosine kinase, is present in around $22 \%$ of early breast cancers, $35 \%$ of locally advanced and metastatic tumours, and $40 \%$ of inflammatory breast cancers, and is associated with aggressive disease and poor prognosis (Ross et al., 2009). Patients with HER2-positive locally advanced or inflammatory breast cancer are therefore in particular need of effective treatment. Trastuzumab (Herceptin, Roche, Basel, Switzerland), a recombinant humanized monoclonal antibody that targets HER2, has efficacy as monotherapy (Baselga et al., 2005) and improves results of chemotherapy in patients with HER2-positive metastatic (Slamon et al., 2001; Marty et al., 2005) and early operable breast cancer (Smith et al., 2007; Romond et al., 2005; Slamon et al., 2005). It is widely approved for use as monotherapy and in combination with chemotherapy or hormone therapy in these patients, but not specifically in those with locally advanced or inflammatory breast cancer. In a pilot study, anthracycline and paclitaxel were successfully combined with trastuzumab in patients with metastastic disease (Bianchi et al., 2003). To reduce the risk of cardiac toxic effects, only three cycles of doxorubicin were given in the pilot study, which corresponds to a cumulative dose of $180 \mathrm{mg}$ per $\mathrm{m}^{2}$ of body surface area (Gianni et al., 2009). No patient developed symptomatic cardiac dysfunction, although four patients (of 16) had reversible asymptomatic decreases in left ventricular ejection fraction to $50 \%$ or lower.

The neoadjuvant Herceptin (NOAH) study was designed to assess efficacy of neoadjuvant chemotherapy with trastuzumab followed by adjuvant trastuzumab versus neoadjuvant chemotherapy alone in patients with HER2-positive locally advanced or inflammatory breast cancer. The NOAH study randomized 228 patients with centrally confirmed HER2+ locally advanced breast cancer to a chemotherapy regimen consisting of 3 cycles of doxorubicin plus paclitaxel (AT); 4 cycles of paclitaxel (T); and 3 cycles of cyclophosphamide, methotrexate, and fluorouracil (CMF), with and without trastuzumab. The addition of trastuzumab significantly improved overall response rate $(81 \%$ vs $73 \%, \mathrm{P}=0$. 18 ) and pCR rates ( $43 \%$ vs $23 \%, P=0,002)$ (Gianni et al., 2010).

The primary objective was to compare event-free survival, which was defined as time from randomization to disease recurrence or progression (local, regional, distant, or contralateral) or death from any cause, in patients with HER2-positive disease treated with and without trastuzumab.

Trastuzumab significantly improved event-free survival in patients with HER2-positive breast cancer (3-year event-free survival 71\% [95\% CI 61-78; $n=36$ events] with trastuzumab, vs 56\% [46-65; n-51 events] without; hazard ratio 0.59 [95\% CI 0-38-0-90]; p-0.013). Trastuzumab was well tolerated and, despite concurrent administration with doxorubicin, only two patients $(2 \%)$ developed symptomatic cardiac failure. Both responded to cardiac drugs.

The results of the NOAH study have shown that in patients with HER2-positive locally advanced or inflammatory breast cancer, addition of 1 year of trastuzumab (starting as neoadjuvant and continuing as adjuvant therapy) to neoadjuvant chemotherapy improved overall response rates, almost doubled rates of pathological complete response, and reduced risk of relapse, progression, or death compared with patients who did not receive 
trastuzumab. Investigators recorded a benefit of trastuzumab in all subgroups tested, including women with inflammatory disease (27\% of HER2- positive patients) who benefited substantially from trastuzumab.

The results of the NOAH study consolidate those of other studies of trastuzumab in the neoadjuvant setting. In these mainly non-randomised studies, pathological complete response rates (variously defined) ranged from $17 \%$ to $73 \%$, and were better than they were in historical' or concurrent HER2-negative controls (Gluck et al., 2008; Untch et al., 2008). One randomised trial in patients with operable non-inflammatory disease was stopped early when the pathological complete response rate in the trastuzumab group was more than twice as high as that of the control group (65\% vs 26\%) (Buzdar et al., 2005). Patient numbers in this study were small, but preliminary results from another randomized study also show a doubling in pathological complete response rate in the trastuzumab group. These response rates to primary systemic therapy are a surrogate for relapse-free and overall survival in patients who were unselected for HER2 status.

Despite concurrent use of doxorubicin, paclitaxel, and trastuzumab in the NOAH trial, incidence of symptomatic cardiac failure was low $(<2 \%)$ and less than was expected $(2.8-$ $4.1 \%)$ on the basis of adjuvant trials in which trastuzumab was given concurrently with paclitaxel after completion of doxorubicin and when trastuzumab was given as monotherapy after completion of a range of cytotoxic regimens (2\%). These findings support the accumulating evidence that trastuzumab can be given concurrently with anthracyclines with a low frequency of symptomatic cardiac dysfunction, provided that low cumulative doses or less cardiotoxic anthracyclines are used, and careful cardiac monitoring is done.

The addition of trastuzumab to neoadjuvant sequential anthracycline-taxane chemotherapy (with and without capecitabine) was also investigated in the phase III GeparQuattro study, and led to a doubling of pCR rates (31.8\% vs $15.4 \%, \mathrm{P}<0.001)$ (Von Minckwitz et al., 2008). With the emergence of lapatinib (Tykerb), a dual tyrosine kinase inhibitor against HER1 and HER2, the CALGB is conducting a randomized phase III trial to evaluate paclitaxel with trastuzumab or lapatinib, or both in the preoperative setting. Several other trials are ongoing to evaluate these 2 drugs in the neoadjuvant setting, including Neo-ALTTO (Neoadjuvant Lapatinib and/or Trastuzumab Treatment Optimization) in phase III and CHERLOB in phase II.

Trastuzumab $(\mathrm{H})$ in combination with chemotherapy improves outcomes in patients with HER2-positive breast cancer and is integral to the standards of care for these patients. However, in some patients disease progression still occurs. Pertuzumab $(\mathrm{P})$ and trastuzumab $(\mathrm{H})$ target different epitopes of HER2, and their use in combination has demonstrated improvement in response rates. NEOSPHERE study (Gianni et al., 2011) assessed the efficacy and safety of pertuzumab added to trastuzumab-based neoadjuvant chemotherapy in women with HER2-positive operable, locally advanced/inflammatory breast cancer who had not received prior cancer therapy.

Patients $(n=417)$ with HER2-positive (IHC3+ or IHC2+ and FISH/CISH+) breast cancer were randomized 1:1:1:1 to receive 4 neoadjuvant cycles of docetaxel (T) plus H, THP, HP or TP. Pertuzumab (P) was given at a loading dose of $840 \mathrm{mg}$ and $420 \mathrm{mg}$ maintenance, trastuzumab $(\mathrm{H})$ at a loading dose of $8 \mathrm{mg} / \mathrm{kg}$ and $6 \mathrm{mg} / \mathrm{kg}$ maintenance, and docetaxel $(\mathrm{T})$ at $75 \mathrm{mg} / \mathrm{m}^{2}$ with escalation to $100 \mathrm{mg} / \mathrm{m}^{2}$ if tolerated in a 3weekly schedule. The primary endpoint was $\mathrm{pCR}$ in the breast. 
About $40 \%$ of patients had locally advanced/inflammatory breast cancer and approximately $50 \%$ were ER/PR negative. THP combination (docetaxel + trastuzumab + pertuzumab) significantly improved the pCR rate compared with $\mathrm{TH}$ (docetaxel + trastuzumab) alone: $45.8 \%$ (95\% CI 36.1-55.7) vs $29.0 \%$ (95\% CI 20.6-38.5), $\mathrm{p}=0.0141$. Patients receiving THP (docetaxel + trastuzumab + pertuzumab) had the highest pCR rate regardless of ER/PR status, although the greatest treatment benefit in all 4 arms was observed in ER/PR-neg patients. The chemotherapy-free HP (trastuzumab+pertuzumab) arm achieved a pCR rate of $16.8 \%$. THP (docetaxel + trastuzumab + pertuzumab) had a similar safety profile to TH. The incidence of AEs was lowest in the HP (trastuzumab+pertuzumab) arm.

Thus, the addition of pertuzumab to trastuzumab-based neoadjuvant chemotherapy resulted in a significant improvement of the $\mathrm{pCR}$ rate with no new safety signals of concern. Pertuzumab and trastuzumab have complementary mechanisms of action as pertuzumab inhibits HER2:HER3 heterodimerisation, thereby providing a potential mechanism to overcome tumour escape. These results support the rationale for a planned Phase III, double-blind, placebo-controlled trial evaluating pertuzumab added to standard trastuzumab-based therapy in women with HER2- positive breast cancer.

Despite the dramatic improvement in the outcome of HER2+ breast cancers since the widespread use of HER2-directed therapies, such as trastuzumab, patients continue to develop recurrences and disease progression. The mechanisms of intrinsic and acquired resistance to trastuzumab are likely multifactorial and are being exploited by the use of novel targeted agents in clinical development. The phosphoinositide-3-kinase (PI3K) pathway plays a key role in resistance to trastuzumab through increased signaling through upstream growth factor receptors, PTEN mutations, and other mechanisms, and therefore, is an excellent target for drug development in patients with trastuzumab-resistant, HER2+ breast cancers. Available clinical trials demonstrate encouraging activity of mTOR inhibitors in combination with trastuzumab monotherapy or trastuzumab-based chemotherapy in patients with HER2 ${ }^{+}$metastatic breast cancer pretreated with trastuzumab with or without lapatinib. The results of early-stage clinical trials are currently being confirmed in 2 large phase III trials (Brachman et al., 2009; Vazguez-Martin et al., 2009). Other agents, targeting the PI3K pathway, are in early clinical development for HER2+ breast cancers.

Cross-talk between the estrogen receptor (ER) and the phosphoinositide-3-kinase (PI3K)/Akt/mammalian target of rapamycin (mTOR) pathways is a mechanism of resistance to endocrine therapy, and blockade of both pathways enhances antitumor activity in preclinical models. Study of Baselga et al.(2009) explored whether sensitivity to letrozole was enhanced with the oral mTOR inhibitor, everolimus (RAD001). Response rate by clinical palpation in the everolimus arm was higher than that with letrozole alone (ie, placebo; $68.1 \% \mathrm{v} 59.1 \%$ ), which was statistically significant at the preplanned, onesided, $\mathrm{a}=0.1$ level $(\mathrm{P}=0.062)$. Marked reduction in progesterone receptor and cyclin D1 expression occurred in both treatment arms, and dramatic downregulation of phosphorS6 occurred only in the everolimus arm. An antiproliferative response, as defined by a reduction in Ki67 expression to natural logarithm of percentage positive Ki67 of less than 1 at day 15, occurred in $52(57 \%)$ of 91 patients in the everolimus arm and in $25(30 \%)$ of 82 patients in the placebo arm $(\mathrm{P}<0.01)$.

The exact mechanism by which mTOR inhibitors appear to reverse resistance to trastuzumab remains unclear. Future clinical trials should attempt to delineate these mechanisms so that patients can be selected appropriately for these therapeutic approaches. 


\subsection{Triple-negative breast cancer}

Triple-negative (ER-negative, PgR-negative, and HER2 receptor-negative) breast cancers (TNBC) account for approximately $15 \%$ of all breast cancers and, though in and of itself it is a heterogeneous group, it often exhibits an aggressive phenotype with a generally poor prognosis. Unlike HER2+ or hormone receptor- positive breast cancers, triple-negative tumors lack an established therapeutic target and though initially responsive to many standard treatment regimens, progression and recurrence can be rapid and refractory to alternative approaches. Loss or inactivation of breast cancer type 1 (BRCA1) leads to defects in certain DNA repair pathways. Most BRCA1 mutant breast cancers lack ER, PgR, and HER2 expression, and this association has raised the question of defective BRCA1 function in sporadic (non-familial) TNBC (Sorlie et al., 2003). This led to the hypothesis that triple-negative tumors may be more sensitive to DNA damaging agents, such as platinums. A retrospective analyses of patients with triple-negative breast cancer who received taxane/ platinum-based primary chemotherapy demonstrated an overall response of $39 \%$ (Uhm et al., 2009), while studies of platinum monotherapy or combinations in the neoadjuvant setting have produced pCR rates of $22 \%-50 \%$ (Garber et al., 2006; Chang et al., 2008).

To exploit the defective DNA repair mechanisms in triple-negative and BRCA-deficient breast cancers, recent trials investigated the effect of interfering further with DNA repair through the use of novel small molecule inhibitors of poly-ADP ribose polymerase (PARP). This is a critical enzyme in cell proliferation and DNA repair. Results from several preliminary trials have been reported. The first was a phase II trial which evaluated the oral PARP inhibitor, olaparib, as a single agent as second- or later-line therapy in 54 patients with locally advanced or metastatic BRCA-deficient breast cancer (Tutt et al., 2009). Despite this use of olaparib as a single agent in a pretreated population, a response rate of $41 \%$ was reported for patients receiving the higher of 2 evaluated doses.

One of the key issues related to interpretation of trials investigating triple-negative breast cancer is the heterogeneity of this tumor subtype. Although most basal-like tumors are also triple-negative, there is discordance between triple-negative designation on clinical assays and basal-like breast cancer on gene expression arrays (Schneider et al., 2008). There is also heterogeneity within triple-negative breast cancer regarding expression of p53, BRCA1, and other relevant genes. Thus, there is danger in making clinical decisions based on cross- trial comparisons, as the patient populations are not identical and the definition of triple-negative breast cancer or basal-like breast cancer differs across studies. Additionally, subset analyses with non-centralized review of tumor markers should be interpreted with caution since a substantial percentage of patients may not have triplenegative disease based on incorrect classification. Prospective trials with carefully defined triple-negative status using validated biomarker analysis are necessary to optimize the use of targeted therapy in this patient population.

\subsection{Molecular profiling in prognosis and patient selection for neoadjuvant systemic therapy}

Gene expression profiling with the use of DNA microarrays has added valuable information to our understanding of breast cancer biology. In the seminal work of Perou et al. (2011) the ability to interrogate thousands of genes at the same time was translated into a "molecular portrait" of each tumor sample studied, and the concomitant analysis of the individual 
molecular portraits of breast cancer tumor samples made the definition of molecular subtypes of breast cancer possible (Perou et al., 2011). In order to analyze this large quantity of information (thousands of genes per sample evaluated), a hierarchical clustering method was used to group genes according to similar patterns of expression. The proposed molecular classification of breast cancer was divided into five classes: luminal-A, luminal-B, basal-like, HER2-positive and normal-like tumors (Sotiriou et al., 2003; Sorlie et al., 2003). Subsequently, the correlation between molecular subtypes and clinical data have shown a significant difference in overall survival between the subtypes.

Despite this progress, the clinical applicability of molecular classification is limited by the tight correlation between the molecular subtypes and currently available immunohistochemical markers (ER, PR, HER2, Ki67) (Sotiriou \& Pusztai, 2009). For example, the molecular subtype HER2-positive is clinically detected by IHC or fluorescent in situ hybridization (FISH) according to published guidelines (Sauter et al., 2009). Although a good correlation has been established between the molecular subtype HER2 and clinically assessed HER2-positive breast cancer, the opposite is not true, because 30\% of HER2-positive breast cancers are molecularly characterized as luminal-B (Cheang et al., 2009). Luminal-A and luminal-B molecular subtypes are, by definition, hormone receptor positive tumors, but the distinction between these two subtypes is controversial.

One of the proposed clinical definitions characterizes luminal-A and luminal-B tumors using hormone receptor status, HER2 status and the Ki67 index (percentage of Ki67-positive nuclei by IHC). Luminal-A is defined as being ER- and/or PR-positive, HER2-negative and Ki67-low (Ki67 index < 14\%). Luminal-B is defined as ER- and/or PR- positive, HER2negative and Ki67-high (Ki67 index > 14\%). Another luminal-B subtype has also been proposed, namely luminal HER2 enriched, with tumors being ER- and/or PR- positive, HER2-positive and Ki67-high (ki67 index > 14\%) (Perou, 2011).

Study Jinno et al (2011) was to evaluate the clinical utility of breast cancer intrinsic subtypes in the prediction of pathological complete response (pCR) in a cohort of breast cancer patients receiving neoadjuvant chemotherapy.

Patients with stage II/III breast cancer received 4 cycles of chemotherapy XT (capecitabine $1650 \mathrm{mg} / \mathrm{m}^{2}$ on days $1-14$ and docetaxel $60 \mathrm{mg} / \mathrm{m}$ on day 8 every 3 weeks), followed by 4 cycles of FEC (fluorouracil $500 \mathrm{mg} / \mathrm{m}^{2}$, epirubicin $90 \mathrm{mg} / \mathrm{m}^{2}$, cyclophosphamide $500 \mathrm{mg} / \mathrm{m}^{2}$ ). Immunohistochemical (IHC) analysis of ER, PgR, HER2. EGFR, cito-ceratine 5/6. and Ki67 was performed in core needle biopsy samples at baseline. Tumors were classified as luminal A (ER+ and/or PgR+, and Ki67<20\%), Luminal B (ER+ and PgR+, and Ki67 > 20\%). Luminal-HER2 (ER+ and/or PgR+, and HER2+), HER2-enriched (ER- PgR-, and HER2+), or triple-negative (ER-, PgR-, and HER2-). Triple-negative tumors with and without EGFR+ and/or cito-ceratine 5/6+ were further classified as basal-like and nonbasal-like TN (NBTN), respectively. Pathologic complete response (pCR) was defined as no microscopic evidence of residual viable tumor cells, invasive or noninvasive, in all resected specimens of the breast. Twenty-six (31.3\%) patients were classified as luminal A, $12(14.5 \%)$ were luminal B, 15 (18.1\%) were luminal-HER2, 9 (10.8\%) were HER2, 10 (12.0\%) were basallike, and $11(13.3 \%)$ were NBTN. The overall response rate was $90.4 \%$, including a complete response in 30 patients and a partial response in 45 patients. The overall pCR rate was $15.5 \%$ $(12 / 83)$. The highest pCR rate $(40.0 \%)$ was observed in patients with basal- like tumors. In triple-negative patients, basal-like patients showed significantly higher $\mathrm{pCR}$ rate than NBTN patients $(40.0 \%$ vs. $9.1 \%$. $\mathrm{p}=0.01)$. There were no cases with $\mathrm{pCR}$ in a cohort of luminal- 
HER2 subtype patients. A higher proportion of luminal B patients had pCR than luminal A patients $(25.0 \%$ vs. $3.8 \%, \mathrm{p}=0.01)$. Data indicate that breast cancer subtypes are useful predictive biomarkers of $\mathrm{pCR}$ in breast cancer patients treated with neoadjuvant systemic chemotherapy.

Despite advances, $20 \%$ to $30 \%$ of patients with early breast cancers will experience relapse with distant metastatic disease. Risk of recurrence is influenced by stage at initial presentation and the underlying biology of the tumor. Tumor size, nodal involvement, grade, lymphovascular invasion, and estrogen receptor (ER) and human epidermal growth factor receptor 2 (HER2) status are all independent risk factors for relapse (Chia et al., 2008). However, we do not have a comprehensive understanding of the patterns of spread and specific sites of recurrence.

The objectives of the study by Kennecke et al. (2010) were to determine the influence of breast tumor molecular subtypes on site of metastatic disease and to define the associated patient outcomes using a large validated tissue microarray (TMA) of primary invasive breast cancer specimens. Ten-year survival estimates were significantly different ( $P$ $<0.001$ ) among subgroups; $70 \%$ of patients with luminal A tumors were alive at 10 years compared with $54.4 \%$ of luminal B, $46.1 \%$ of luminal/ HER2, 48.1\% of HER2-enriched, $52.6 \%$ of basal-like, and $62.6 \%$ of nonbasal triple negative (TN) patients. Median duration of survival from time of first distant metastasis also differed significantly, with luminal A patients achieving the longest survival (2.2 years) followed by luminal B (1.6 years), luminal/HER2+ (1.3 years), HER2-enriched (0.7 years), basal- like (0.5 years), and triplenegative $(\mathrm{TN})$ nonbasal patients $(0.9$ years; $\mathrm{P}<0$.001). These differences in relapse according to subtype were maintained with 15-year distant relapse rates for luminal A (27.8\%), luminal B (42.9\%), luminal/HER2 (47.9\%), HER2-enriched (51.4\%), basal-like $(43.1 \%)$ and TN nonbasal (35.1\%) subgroups.

The study by Kennecke et al. (2010) demonstrates important differences in metastatic behavior between the breast cancer subtypes as defined by a panel of immunohistochemical markers and contributes to an expanding knowledge of prognostic and predictive markers that will allow individualized therapy for advanced breast cancer similar to current approaches in development for early-stage disease.

In the article by Voduc et al. (2010) a six-marker immunohistochemical panel (ER, PR, HER2, epidermal growth factor receptor, CK 5/6, and Ki-67) was used to classify nearly 3,000 patients treated with breast conserving surgery (BCS) and radiation therapy (RT) or mastectomy as luminal-A, luminal-B, luminal-HER2, HER2-enriched, basal-like, and triplenegative nonbasal. The intrinsic molecular subtype was successfully determined in 2,985 tumors. The median follow-up time was 12 years, and there have been a total of 325 local recurrences and 227 regional lymph node recurrences. Luminal A tumors (ER or PR positive, HER2 negative, Ki- $67<14 \%$ ) had the best prognosis and the lowest rate of local or regional relapse. For patients undergoing breast conservation, HER2-enriched and basal subtypes demonstrated an increased risk of regional recurrence, and this was statistically significant on multivariable analysis. After mastectomy, luminal B, luminal-HER2, HER2enriched, and basal subtypes were all associated with an increased risk of local and regional relapse on multivariable analysis.

In a second important study assessing molecular profiling using an alternative classification scheme for risk assessment of locoregional relapse (LRR) in breast cancer, Mamounas et al. (2010) evaluated the 21-gene profile (Oncotype DX), in more than 1,500 patients with node- 
negative ER-positive disease from National Surgical Adjuvant Breast and Bowel Project studies treated by breast-conserving surgery (BCS) plus radiotherapy (RT) or mastectomy without RT. In the patients treated with mastectomy, a high recurrence score (RS) was associated with a significantly higher risk of local-regional recurrence (LRR). The results demonstrated that patients with node-negative ER-positive disease and a high 21 -gene recurrence score, particularly those patients younger than 50 years, had a relatively high risk of LRR with mastectomy without radiotherapy (RT), suggesting that the 21-gene profile test may identify a cohort of patients with lymph node-negative disease who may potentially benefit from postmastectomy RT. Given that this study was a retrospective analysis, it will be important to validate these data in another data set before changing standard treatment recommendations. In contrast, it appears that the low LRR rate in ERpositive patients with a low 21-gene recurrence score is comparable to the low LRR rate in the luminal-A group identified in the Voduc et al. (2010) study and it is likely that a majority of these patients with low recurrence scores have tumors that would be categorized as luminal-A tumors.

In addition to larger validation studies and companion molecular protocols linked to clinical trials evaluating molecular profiling in locoregional management of breast cancer, there is a need for additional basic research to identify molecular profiles that consistently predict for locally aggressive disease. It should be noted that both classification schemes (the 21-gene recurrence score and the luminal/HER2/basal scheme) are derived from years of research demonstrating the potential of these markers in risk assessment for metastasis and overall survival. While locoregional relapse and systemic metastasis are clearly linked, risk factors and potential molecular profiles, which best predict for locoregional relapse, may be quite different from those molecular profiles that predict for systemic metastasis.

It appears from both the Voduc et al. (2010) study and the Mamounas et al. (2010) study that patients with favorable luminal-A tumors or those with a low 21-gene recurrence score are at low risk for both local relapse and systemic disease. Patients with basal-like tumors, which are clearly at high risk for systemic metastasis, may also have an increased risk for local-regional recurrence (LRR) after surgical and radiotherapy (RT) treatment.

Although the proposed classification allows for broader application, due to the widespread use of IHC, some inherent limitations raise concern: IHC evaluation is limited by interobserver variability, qualitative readouts and technical reproducibility (Oyama et al., 2007).

\subsection{Prognostic utility of multigene assays}

The 21-gene recurrence score (RS) assay and 70-gene signature demonstrated prognostic utility in patients with both node-negative and node-positive early-stage, hormone receptor-positive breast cancer (Sotiriou \& Pusztai, 2009). Neither assay has been validated nor demonstrated prognostic utility in hormone receptor-negative breast cancer. Both the 21-gene and 70-gene assays also provide additional information for treatment decision-making beyond algorithms based on standard clinicopathologic criteria such as Adjuvant-Online (Albain et al., 2009). Although the original validation of the 21-gene RS assay established its prognostic ability in patients treated with adjuvant tamoxifen, a recent study demonstrated similar prognostic ability in patients who received an aromatase inhibitor as upfront adjuvant therapy (Dowsett et al., 2008). For both prognosis and prediction, only the 21-gene RS assay has been studied with specimens from phase III adjuvant therapy trials (Albain et al., 2009, 2010). 
The ability of Genomic Grade Index (GGI) to predict response to neoadjuvant chemotherapy was evaluated in 229 tumor samples collected before neoadjuvant chemotherapy with paclitaxel, fluorouracil, doxorubicin and cyclophosphamide (T/FAC) (Liedtke et al., 2009). In general, pathologic complete response (pCR) is associated with better disease outcome regardless of hormone receptor status (Guarneri et al., 2006). Histologic grade is known to be a predictor of pathologic complete response, but inherent limitations to histological grade assessment limits its applicability.

In the evaluation of GGI as a predictor of response, a more precise method for evaluating pathologic response called residual cancer burden (RCB) was used as a comparator (Symmans \& Peintiger, 2007). RCB better defines different ranges of pathologic response after neoadjuvant chemotherapy. It is calculated as continuous variable, using pathologic measurements of the primary tumor and nodal metastases. In post-treatment surgical resection specimens a bidimensional diameter of primary tumor bed and the proportion of invasive tumor cells in the same area are measured. The number of nodes containing metastases and the diameter of the large lymph node metastases are also components of RCB. The prognostic information obtained with RCB was evaluated in 382 patients treated with neoadjuvant chemotherapy. In a multivariate analysis containing age, clinical stage, hormone receptor status, hormone treatment and pathologic response (pCR versus residual disease), RCB was an important prognostic factor associated with distant relapse-free survival $(\mathrm{HR}=2.50 ; 95 \%$ CI 1.70-3.69; $\mathrm{p}<0.001)$. Minimal residual cancer burden (RCB-I) and pCR (RCB-0) were associated with similar favourable long-term relapse-free prognosis. $\mathrm{RCB}$ adds to a better understanding of response to primary chemotherapy.

In the evaluation of GGI compared to RCB to predict chemotherapy response, a data set comprising 229 samples from 132 ER-positive and 97 ER-negative patients was used. All patients had HER2 non-amplified tumors, which avoided the interference of chemotherapy and trastuzumab response. Pathologic response was assessed as follows: RCB- 0 indicating pCR, and RCB-I, RCB-II, RCB-III for minimal, intermediate and extensive residual disease respectively. GGI was assessed for each tumor sample and assigned as low or high risk, as in the original publication, but also as a continuous variable. The GGI evaluation characterized $84.6 \%$ of grade 1 tumors as low risk and $88.3 \%$ of grade 3 as high risk. The histological grade 2 group was divided into $62.7 \%$ low risk and $37.3 \%$ high risk. For the ERpositive and ER-negative subgroups, $44.8 \%$ and $89.6 \%$ respectively were assigned to the GGI high-risk category. For the overall group treated with neoadjuvant T/FAC, high-risk GGI was associated with higher response than low-risk GGI $(40 \%$ versus $12 \%$; $<<0.001)$. A positive correlation was observed between GGI high-risk category and the level of observed response to neoadjuvant chemotherapy, with $85.8 \%$ of patients with RCB-0 or RCB-I, characterized as GGI high-risk (Metzger et al., 2010).

The similar outcome predictions between the different signatures motivated a search for underlying biologic processes that could be represented by different genes in nonoverlapping signatures. A large meta-analysis of publicly available breast cancer gene expression and clinical data evaluated the contribution of known biological processes to the performance of different gene signatures. "Coexpression modules" for ER signaling, ERBB2 amplification and proliferation were generated, putting together a comprehensive list of genes with highly correlated expression (Metzger et al., 2010). The meta-analysis was able to confirm in 2833 patients that the initial classification of breast cancer 
molecular subtypes was highly conserved, with the exception of normal-like breast cancer, which could not be identified.

In all breast cancer subtypes (HER2, basal-like, luminal-A, luminal-B) the coexpression module proliferation was the most important determinant of prognosis. Although the coexpression module HER2 could be identified in the subtype HER2, the prognostic information was mainly driven by genes related to proliferation. HER2 and basal-like subtypes were consistently characterized as high proliferative tumors. In the luminal subtypes, the module of genes related to proliferation could divide this group into a lowproliferative subtype (A) with better prognosis and a highly proliferative group with poorer prognosis (B). The evaluation of clinical variables demonstrated that tumor size and nodal status still have independent prognostic value and need to be evaluated together with the information obtained from gene-signatures.

Understanding breast cancer molecular heterogeneity has made it possible to develop gene signatures that can be applied to predict prognosis and response to therapies in daily practice. The superiority of gene signatures to classic histopathologic variables is related to their ability to better define a greater proportion of low-risk patients that do not need to be treated with systemic neoadjuvant and adjuvant therapy, while still correctly identifying those patients who fall into a high-risk group. Clinical variables related to the measurement of tumor progression such as tumor size and nodal involvement remain significantly associated with prognosis and should therefore continue to be evaluated in conjunction with gene signatures.

\section{Conclusion}

Neoadjuvant systemic therapy is an appropriate management strategy in certain welldefined patient cohorts, namely those in whom surgery is not feasible due to locally advanced disease at presentation; patients with large tumors requiring mastectomy, but wishing for breast conservation; and patients participating in clinical trials. Neoadjuvant therapy does not improve the outcomes in terms of disease-free survival or overall survival (OS), compared with adjuvant systemic treatment, and should not be chosen with this intent. There is currently no evidence to support deviating from a planned neoadjuvant regimen by changing drugs based on the observed response.

Advances in the understanding of breast cancer tumor biology have greatly increased the assessment of patient prognosis, as well as which patients most benefit from neoadjuvant chemotherapy or endocrine therapy. The current challenge is how to best merge multigene assays with clinical biologic variables to achieve classifiers with even greater predictive utility. The enhanced knowledge of breast cancer biologic heterogeneity has also led to the development and clinical investigation of novel therapeutics aimed at cell function and essential signaling pathways. These agents are already producing survival benefits in patients with early-stage or advanced disease. However, many questions remain regarding the appropriate use of these compounds, including optimal patient selection, preventing and overcoming resistance, and management of associated toxicities. Furthermore, there is increased attention to the need to block multiple targets simultaneously to optimize response and overcome resistance that results from signaling pathway cross-talk.

Finally, multiple biomarkers of response have been evaluated and show promise, but are not yet ready for routine clinical use. 


\section{Acknowledgment}

We thank Dr. Garik Dashan for the preparation of the references.

\section{References}

Albain, K., Paik, S. \& van't Veer, L. (2009). Prediction of adjuvant chemotherapy benefit in endocrine responsive, early breast cancer using multigene assays. Breast; Vol. 18 (suppl 3), pp.141-145, ISSN 0960-9766.

Albain, K., Barlow, W. \& Shak, S. (2010). Prognostic and predictive value of the 21-gene recurrence score assay in postmenopausal women with node-positive, oestrogenreceptor-positive breast cancer on chemotherapy: a retrospective analysis of a randomized trial. Lancet Oncol, Vol. 11, pp. 55-65, ISSN 1470-2045.

Albain, K., Carey, L., Gradishar, W., Gralow, J. \& Lipton, A. (2010). Proceedings of the First Global Workshop on Breast Cancer : Pathways to the Evalution and Clinical Development of Novel Agents for Breast Cancer. Clinical Breast Cancer, Vol. 10 (N6 December), pp. 421 - 439, ISSN 1526-8209.

Baselga, J., Carbonell, X. \& Castaneda-Soto, N. (2005). Phase II study of efficacy, safety, and pharmacokinetics of trastuzumab monotherapy administered on a 3-weekly schedule. J Clin Oncol, Vol. 23, pp. 2162-2171, ISSN 0732-183x

Baselga, J., Semiglazov, V., Van Dam, P., Manikhas, A. \& Bellet, M. (2009). Phase II Randomized Study of Neoadjuvant Everolimus Plus Letrozole Compared With Placebo Plus Letrozole in Patients With Estrogen Receptor-Positive Breast Cancer. Journal of Clinical Oncology, Vol 27, No 16 (June 1), pp. 2630-2637, ISSN 0732-183x

Bear, H., Anderson, S. \& Smith, R. (2006). Sequential preoperative or postoperative docetaxel added to preoperative doxorubicin plus cyclophosphamide for operable breast cancer: National Surgical Adjuvant Breast and Bowel Project Protocol B-27. J. Clin Oncol, Vol. 24, pp. 2019-2027, ISSN 0732-183x

Bianchi, G., Albanell, J. \& Eiermann, W. (2003). Pilot trial of trastuzumab starting with or after the doxorubicin component of a doxorubicin plus paclitaxel regimen for women with HER2-positive advanced breast cancer. Clin. Cancer Res. Vol. 9, pp. 5944-5951, ISSN 1078-0432

Bonadonna, G., Valagussa, P. \& Bramilla, C. (1998). Primary chemotherapy in operable breast cancer: eight-year experience at the Milan Cancer Institute. J Clin. Oncol, Vol. 16, pp. 93-100, ISSN 0732-183x

Brachmann, S., Hofmann, I. \& Schnell, C. (2009). Specific apoptosis induction by the dual $\mathrm{PI} 3 \mathrm{~K} / \mathrm{mTOR}$ inhibitor NVP-BEZ235 in HER2 amplified and PIK3CA mutant breast cancer cells. Proc Natl Acad Sci USA, Vol. 106, pp. 22299-22304, ISSN 0027-8424

Buzdar, A.; Ibrahim, N., Francis, D., Booser, D., Thomas, E. \& Theriault, R. (2005). Significantly higher pathological complete remission(PCR) rate after neoadjuvant therapy with trastuzumab, paclitaxel and epirubicin chemotherapy: Results of a randomized trial in human epidermal growth factor receptor 2-positive operable breast cancer. J.Clin Oncol. Vol. 23. pp.3676-3685, ISSN 0732-183x

Cataliotti, L., Buzdar, A. \& Noguchi, S. (2006). Comparison of Anastrozole versus Tamoxifen as Preoperative Therapy in Postmenopausal Women with Hormone ReceptorPositive Breast Cancer: The Pre-operative «Arimidex» Compared to Tamoxifen (PROACT) Trial. Cancer. Vol. 106, №10, pp. 2095-2103, ISSN 0008-543x 
Chang, H., Slamon, D. \& Gornbein, J. (2008). Preferential pathologic complete response (Pcr) by triple-negative (-) breast cancer to neoadjuvant docetaxel (T) and carboplatin (C.). J. Clin. Oncol., Vol. 26 (15 suppl), p 31s (abstract 604), ISSN 0732-183x

Cheang, M., Chia, S. \& Voduc, D. (2009). Ki67 Index, HER2 Status, and Prognosis of Patients with luminal B Breast Cancer. J Natl Cancer Inst, Vol. 101, pp. 736-50, ISSN 0027-8874

Chia, S., Norris, B. \& Speers, C. (2008). Human epidermal growth factor receptor 2 overexpression as a prognosis factor in a large tissue microarray series of nodenegative breast cancers. J. Clin. Oncol, Vol. 26, pp. 5697-5704, ISSN 0732-183x

Colleoni, M., Viale, G. \& Zahrieh, D. (2004). Chemotherapy is more effective in patients with breast cancer not expressing steroid hormone receptors: a study of preoperative treatment. Clin Cancer Res, Vol. 10, pp. 6622-6628, ISSN 1078-0432.

Colleoni, M., Viale, G., Zahrieh, D., Bottiglieri, L., Gelber, R., Veronesi, P., Balduzzi, A., Torrisi, R., Luini, A., Intra, M., Dellapasqua, S., Cardillo, A., Ghisini, R., Peruzzotti, G. \& Goldhirsch, A. (2008). Expression of ER, RgR, HER1, HER2, and response: a study of preoperative chemotherapy. Ann. of Oncology, Vol. 19, N.3. (March 2008), pp. 465-472, ISSN 0923-7534.

De Laurentis, M., Cancello, G. \& D'Agostino, D. (2008). Taxane-based combinations as adjuvant chemotherapy of early breast cancer: a meta-analysis of randomized trials. J. Clin Oncol, Vol. 26, pp. 44-53, ISSN 0732-183x

Dixon, M. \& Macaskill, J. (2009). Neoadjuvant Endocrine Therapy. ASCO educational book, 2009. pp. 39-43

Dowsett, M., Cuzich, J. \& Wales, C. (2008). Risk of distant recurrence using Oncotype-DX in postmenopausal primary breast cancer patients treated with anastrazole or tamoxifen: a TransATAC study. Presented at: the San Antonio Breast Cancer Symposium. Abstract 53.

Early Breast Cancer Trialists' Collaborative Group (2005). Effects of chemotherapy and hormonal therapy for early breast cancer on recurrence and 15-year survival: an overview of the randomized trials. Lancet, Vol. 365, pp. 1687-1717, ISSN 0140-6736.

Eiermann, W., Paepke, S. \& Appfelstaedt, J. (2001). Preoperative treatment of postmenopausal patients with letrozole: a randomized double-blind multicenter study. Ann Oncol, Vol. 12, pp. 1527-1532, ISSN 0923-7534

Fisher, B., Bryant, J. \& Wolmark, N. (1998). Effect of preoperative chemotherapy on the outcome of women with operable breast cancer. J Clin Oncol, Vol. 16, pp. 2672-2685, ISSN 0732-183x

Garber, JE., Richardson, A. \& Harris, L. (2006). Neo-adjuvant cisplatin (CDDP) in «triplenegative» breast cancer (BC). Breast Cancer Res Treat, Vol. 100 (suppl 1), S 149 (abstract 3074), ISSN 0167-6806.

Gianni, L., Baselga, J., Eiermann, W., Porta, J. \& Semiglazov, V. (2009). Phase III trial evaluating the addition of paclitaxel to doxorubicin followed by cyclophosphamide, metotrexate and fluorouracil as adjuvant or primary systemic therapy: European Cooperative Trial in Operable Breast Cancer. J Clin Oncol, Vol 27, No 15 (May 2009), pp 2474-2481, ISSN 0732-183X

Gianni, L., Eiermann, W., Semiglazov, V., Manikhas, A. \& Lluch, A. (2010). Neoadjuvant chemotherapy with trastuzumab, followed by adjuvant trastuzumab versus neoadjuvant chemotherapy alone, in patients with HER-positive, locally advanced 
breast cancer (the NOAH trial): a randomized controlled superiority trial with a parallel HER2-negative cohort. The Lancet, Vol 375, pp 377-384, ISSN 0140-6736

Gianni, L., Pienkowski, T., Roman, L., Tseng, L. \& Liu, M. (2011). Addition of pertuzumab (p) to trastuzumab $(\mathrm{H})$-based neoadjuvant chemotherapy significantly improves pathological complete response in women with HER2-positive early breast cancer: result of a randomized phase II study (NEOSPHERE). The Breast. Vol 20, suppl 1 (March 2011), pp. 573, ISSN 0960-9776

Gluck, S., McKenna, E. Jr \& Royce, M. (2008). Capecitabine plus docetaxel, with or without trastuzumab, as preoperative therapy for early breast cancer. Int J Med Sci. Vol 5, pp. 341-346. ISSN 1449-1907

Goldhirsch, A., Ingle, J., Gebber, R., Coates, A., Thurlimann, B. \& Senn, H. (2009). Annals of Oncology, Vol. 20, N 4 (June 2009), pp 1133-1144, ISSN 0923-7534

Guaneri, V., Brogilio, K. \& Kau, S. (2006). Prognostic value of pathologic complete response after primary chemotherapy in relation to hormone receptor status and other factors. J Clin Oncol, Vol. 24, pp. 1037-1044, ISSN 0732-183x

Heys, S., Hutcheon, A. \& Sarkar, T. (2002). Neoadjuvant docetaxel in breast cancer: 3-year survival results from the Aberdeen trial. Clin Breast Cancer, Vol. 3, Suppl 2, pp. 6974, ISSN 1526-8209

Jinno, H., Matsuda, S., Sakata, M., Hayashida, T., Takahashi, M. \& Hirose, S. (2011). Differential pathologic response from primary systemic chemotherapy across breast cancer intrinsic subtypes. The Breast, Vol. 20, suppl 1. (March 2011), p. 39, ISSN 0960-9776.

Kaufmann, M., Hortobagyi, G. \& Goldhirsch, A. (2006). Recommendations from an international expert panel on the use of neoadjuvant (primary) systemic treatment of operable breast cancer: an update. J Clin. Oncol. Vol. 24, pp. 1940-1949, ISSN 0732-183x

Kennecke, H., Yerushalmi, R., Woods, R., Chon, U., Cheang, R. \& Voduc, D. (2010). Metastatic Behavior of Breat Cancer Subtypes. J. Clin. Oncol., Vol. 28, N.20 (July 2010), pp. 3271-3272, NSSN 0732-183X

Liedtke, C., Hatzic, C. \& Symmans, W. (2009). Genomic Grade Index is associated with response to chemotherapy in patients with breast cancer. J.Clin.Oncol., Vol. 27, N 19 (Jul 1), pp. 3185-3191, ISSN 0732-183x

Mamounas, E., Tang, G. \& Fisher, B. (2010). Association between the 21-gene recurrence score assay and risk of locoregional reccurrence in node negstive estrogen receptor positive breast cancer Results from NSAB B14 and NSABP B 20. J Clin Oncol, Vol. 28, pp. 1677 1683, ISSN 0732-183x

Marty, M., Cognetti, F. \& Maraninchi, D. (2005). Randomized phase II trial of the efficacy and safety of trastuzumab combined with docetaxel in patients with human epidermal growth factor receptor 2-positive metastatic breast cancer administered as first-line treatment: the M77001 study group. J. Clin.Oncol, Vol. 23, pp. 4265-74, ISSN 0732-183x

Mauri, D., Pavlidis, N. \& Ioannidis, JP. (2005). Neoadjuvant versus adjuvant systemic treatment in breast cancer: a meta-analysis. J.Natl.Cancer Inst. Vol. 97, N.3 (Feb. 2005), pp. 188-194, ISSN 0027-8874 
Metzger, O., Ignatiadis, M. \& Sotirion, C. (2010). Genomic Grade Index: an important tool for assessing breast cancer tumor grade and prognosis. Critical Reviews in Oncology/Hematology, Vol. 74, pp. 1-10, ISSN 1040-8428.

Oyama, T., Isshikawa, Y. \& Hayashi, M. (2007). The effects of fixation, processing and evalution criteria on immunohistochemical detection of hormone receptors in breast cancer. Breast Cancer, Vol 14, pp. 182-188, ISSN 1340-6868

Perou, C. (2011). Molecular classification of breast cancer and its emerging clinical relevance. The Breast, Vol. 20, Suppl. 1 (March 2011), pp. S2-S3, ISSN 0906-9776

Peto, R (2007). Plenary Lecture presented at the San Antonio Breast Cancer Conference, San Antonio. TX. (December 13), http://www.sabcs.org/EnduringMaterials/Index.asp\#webcast

Ring, A., Smith, I. \& Ashley, S. (2004). Oastrogen receptor status, pathological complete response and prognosis in patients receiving neoadjuvant chemotharapy for early breast cancer. Br J Cancer, Vol. 91, pp. 2012-2017, ISSN 0007-0920

Romond, E., Perez, E. \& Bryant, J. (2005). Trastuzumab plus adjuvant chemotherapy for operable HER2-positive breast cancer. N Engl J Med, Vol. 353, pp. 1673-1684, ISSN 1533-4406

Ross, J., Slodkowska, E., Symmans, W., Pusztai, L., Ravdin, P. \& Hortobagyi, G. (2009). The HER- 2 receptor and breast cancer: ten years of targeted anti-HER-2 therapy and personalized medicine. Oncologist, Vol. 14, pp. 320-368, ISSN 1083-7159

Sauter, G., Lee, J. \& Bartlett, J. (2009) Guidelines for human epidermal growth factor receptor 2 testing biologic and methologic consider ations. J Clinic Oncol, Vol. 27, pp. 1323 1333, ISSN 0732-183x

Schneider, B., Winer, E. \& Foulkes, W. (2008). Triple negative breast cancer risk factors to protential targets. Clin Cancer Res, Vol. 14, pp. 8010-8018, ISSN 1078-0432

Scholl, S., Fourquet, A. \& Asselain, B. (1994). Neoadjuvant versus adjuvant chemotherapy in premenopausal patients with tumors considered too large for breast conserving surgery preliminary results of randomised trial S6. Eur J Cancer, Vol. 30A, pp. 645-652

Semiglazov, V., Topuzov, E. \& Bavli, J. (1994). Primary(neoadjuvant) chemotherapy and radiotherapy compared with primary radiotherapy alone in stage IIb-IIIa breast cancer. Ann Oncol, Vol 5, pp. 591-595, ISSN 0923-7534

Semiglazov, V., Kletsel, A. \& Semiglazov, V. (2005) Exemestane (E) vs tamoxifen (T) as neoadjuvant endocrine therapy for postmenopausal women with ER+ breast cancer( T2N1-2,T3N0-1,T4N0M0)[ abstract]. J Clin Oncol ASCO Annual Meeting Proceedings [Post- Meeting Edition], Vol. 223 (16S), p. 530, ISSN 0732-183x

Semiglazov, V., Semiglazov, V., Dashyan, G., Ziltsova, E. \& Ivanov, V. (2007). Phase II randomized trial of primary endocrine therapy versus chemotherapy in postmenopausal patients with estrogen receptor-positiv breast cancer. Cancer, Vol. 110, pp. 244-254, ISSN 0008-543x.

Slamon, D., Leyland-Jones, B. \& Shak, S. (2001). Use of chemotherapy plus a monoclonal antibody against HER 2 for metastatic breast cancer that over expresses HER2. $N$ Engl J Med, Vol. 344, pp. 783-792, ISSN 1533-4406

Slamon, D. \& Eiermann, W. (2005). Phase III randomized trial comparing doxorubicin and cyclophophomide followed by docetoxel( FC-T) with doxorubicin and cyclophophomide followed by docetaxel and trastuzumab (AC-TH) with docetoxel,carboplatin and trastuzumab(TCH) in HER2 positive early breast 
cancer patients: BCIRG 006 study. Breast cancer Res Treat, Vol. 94(suppl 1), S5, ISSN 0067-6806

Smith, I., Heys, S. \& Hutcheon, A. (2002) Neoadjuvant chemotherapy in breast cancer: significantly enhanced response with docetaxel. J Clin Oncol., Vol. 20, pp. 14561466, ISSN 0732-183x

Smith, I. (2004). Anstrozole versus tamoxifen as preoperative therapy for oestrogen receptorpositive breast cancer in postmenopausal women: Combined analysis of the IMPACT and PROACT trials. Presented at 4th European Breast cancer Conference, Hamburg, Germany.

Smith, I., Dowsett, M. \& Ebbs, S. (2005) Neoadjuvant treatment of postmenopausal breast cancer with anastrazole, tamoxifen, or both in combination: The immediate preoperative anastrazole, tamoxifen, or combined with tamoxifen(IMPACT) multicenter double-blind randomized trual. J Clin Oncol, Vol. 23, N 22, pp. 50185116, ISSN 0732-183x

Smith, I., Procter, M. \& Gelber, R. (2007). For the HERA study team 2 year Follow-up of trastuzumab after adjuvant chemotherapy in HER2 - positive breast cancer. A randomized controlled trial. Lanset, Vol. 369, pp. 29-36, ISSN 0140-6736

Sorlie, T., Tibshirani, R. \& Parker, J. (2003) Repeated observation of breast tumor subtypes in independent gene expression data sets. Proc Natl Acad Sci USA, Vol. 100, pp. 84188423, ISSN 0027-8424

Sotiriou, C., Neo, Sy. \& McShane, L. (2003) Breast cancer classification and prognosis based on gene expression profiles from a population-based study. Proc Natl Acad Sci USA, Vol. 100, pp. 10393-10398, ISSN 0027-8424

Sotiriou, C. \& Pusztai, L. (2009). Gene-expression signatures in breast cancer. N. Engl J Med, Vol. 360, pp. 790-800, ISSN 1533-4406

Symmans, W. \& Peintiger, F. (2007) Measurement of residual breast cancer burden to predict survival afyer neoadjuvant chemotherapy. J.Clin Oncol, Vol 25, pp. 4414-4422, ISSN 0732-183x

Tutt, A., Robson, M. \& Garber, J. (2009). Phase II trial of the oral PARP inhibitor olaparib in BRCA-deficient advanced breast cancer. J Clin Oncol, Vol. 27( suppl) 803s (abstract CRA501), ISSN 0732-183x

Uhm, J., Park, Y. \& Yi, S. (2009). Treatment outcomes and clinicopathologic characteristics of triple-negative breast cancer patients who received platinum-containing chemotherapy. Int J Cancer, Vol. 124, pp. 1457-1462, ISSN 0020-7136

Untch, M., Rezai, M. \& Loibl, S. (2008). Neoadjuvant treatment of HER2 overexpressing primary breast cancer with trastuzumab given concomitantly to epirubicin/cyclophosphamide followed by docetaxel \pm capecitabine. First analysis of efficacy and safety of the GBG/AGO multicenter intergroup-study "GeparQuattro". $6^{\text {th }}$ European Breast Cancer Conference. Berlin, Germany, April 1519. Abstr 1LB

Vazguez-Martin, A., Oliveras-Ferreros, C. \& del Barco, S. (2009). m TOR inhibitors and the anti-diabetic biguanide metphormin: new insights into the molecular management of breast cancer resistance to the HER 2 tyrosine kinase inhibitor lapatinib (Tykerb). Clin Transl Oncol, Vol. 11, pp. 455-459, ISSN 1699-048x

Voduc, K., Cheang, MCU. \& Tyldesley, S. (2010). Breast cancer subtypes and the risk of local and regional relapse. J Clin Oncol, Vol. 28, pp. 1684-1691, ISSN 0732-183x 
Von Minckiwitz, G., Rezai, M. \& Loibl, S. (2008). Effect of trastuzumab on pathologic complete response rate of neoadjuvant EC-docetaxel treatment I HER 2overexpressing breast cancer: Results of the phase III Gepar-Qattro study (abstract 226) .ASCO Breast Cancer Symposium.

Von Minckwitz, G., Kummel, S. \& Vogel, P. (2008). Intensified neoadjuvant chemotherapy in early- responding breast cancer: phase III randomized GeparTrio study. I Natl Cancer Inst. Vol. 100, pp. 552-562, ISSN 0027-8874

Von Minckwitz, G., Kummel, S. \& Vogel, P. (2008). Neoadjuvant vinorelbin- capecitobine versus docetaxel-doxorubicin-cyclophosphimide in rarly nonresponsive breast cancer: phase III randomized Gepar Trio. J Natl Cancer Inst. Vol. 100, pp. 542-551, ISSN 0027-8874

Wolmark, N., Wang, J., Mamounas, E., Bryant, J. \& Ficher, B. (2001). Preoperative chemotherapy in patients with operable breast cancer: nine year results from National Surgical Adjuvant Breast and Bowel Project B-18. J Natl Cancer Inst Monogr. Vol. 30, pp. 96-102, ISSN 0027-8874 


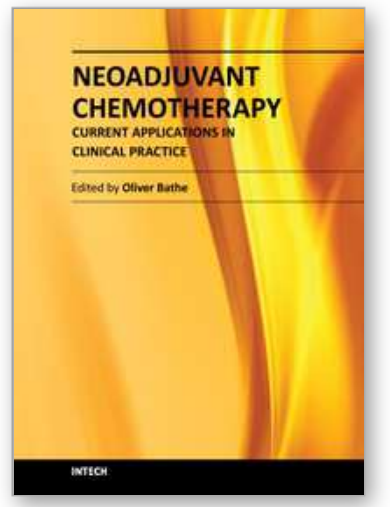

\author{
Neoadjuvant Chemotherapy - Current Applications in Clinical \\ Practice \\ Edited by Dr. Oliver Bathe
}

ISBN 978-953-307-994-3

Hard cover, 268 pages

Publisher InTech

Published online 01, February, 2012

Published in print edition February, 2012

The most significant advances in cancer therapy in recent years have involved the development of systemic therapeutics. With improvements in response rates in solid tumors, opportunities have arisen to enhance the effectiveness of surgery. Administration of systemic therapy prior to surgery - neoadjuvant chemotherapy represents one approach by which clinicians have successfully reduced the extent of surgery and, in some instances, positively impacted on clinical outcomes. This collection of works by expert clinicians from a variety of disciplines represents an exploration of the current knowledge of the role of neoadjuvant chemotherapy in diverse tumor types.

\title{
How to reference
}

In order to correctly reference this scholarly work, feel free to copy and paste the following:

Vladimir F. Semiglazov and Vladislav V. Semiglazov (2012). Neoadjuvant Systemic Therapy in Breast Cancer, Neoadjuvant Chemotherapy - Current Applications in Clinical Practice, Dr. Oliver Bathe (Ed.), ISBN: 978-953307-994-3, InTech, Available from: http://www.intechopen.com/books/neoadjuvant-chemotherapy-currentapplications-in-clinical-practice/neoadjuvant-systemic-therapy-in-breast-cancer

\section{INTECH}

open science | open minds

\section{InTech Europe}

University Campus STeP Ri Slavka Krautzeka 83/A 51000 Rijeka, Croatia Phone: +385 (51) 770447

Fax: +385 (51) 686166 www.intechopen.com

\section{InTech China}

Unit 405, Office Block, Hotel Equatorial Shanghai No.65, Yan An Road (West), Shanghai, 200040, China 中国上海市延安西路65号上海国际贵都大饭店办公楼 405 单元 Phone: +86-21-62489820

Fax: $+86-21-62489821$ 
(C) 2012 The Author(s). Licensee IntechOpen. This is an open access article distributed under the terms of the Creative Commons Attribution 3.0 License, which permits unrestricted use, distribution, and reproduction in any medium, provided the original work is properly cited. 